\title{
Invasion of Adjacent Lumbar Vertebral Body from Renal Pelvis Carcinoma: Associated With Bone Metastasis But Easily Overlooked on Initial CT Scan
}

\author{
TZU-YAO LIAO ${ }^{1}$, CHUANG-CHI LIAW $^{1}$, KE-HUNG TSUI ${ }^{2}$ and YU-HSIANG JUAN ${ }^{3}$ \\ ${ }^{1}$ Division of Hemato-Oncology, Department of Internal Medicine, Chang-Gung Memorial \\ Hospital and Chang-Gung University College of Medicine, Taoyuan, Taiwan, R.O.C.; \\ ${ }^{2}$ Department of Urology, Chang-Gung Memorial Hospital and Chang-Gung \\ University College of Medicine, Taoyuan, Taiwan, R.O.C.; \\ ${ }^{3}$ Department of Medical Imaging and Intervention, Chang-Gung Memorial Hospital \\ and Chang-Gung University College of Medicine, Taoyuan, Taiwan, R.O.C.
}

\begin{abstract}
Background/Aim: We hypothesized that regional tumor growth into L1 and L2 vertebral bodies from renal pelvis carcinoma was linked to the development of bone metastases. Materials and Methods: Criteria for the study were: (i) Metastatic renal pelvis carcinoma confirmed via pathology and computed tomographic (CT) scan, (ii) L1 and L2 invasion confirmed from retrospective CT scan review, and (iii) detection of bone metastases using radionuclide images/CT scans. Results: A total of 71 cases were enrolled in the study. Initial L1 and L2 vertebral body invasion. were detected in 45 (63\%) patients. As well as L1 and L2 invasion, 32 (71\%) had development of bone metastases. All bone lesions were osteolytic. Initial L1 and L2 invasion $(p<0.00001)$ was associated with the development of bone metastasis. Conclusion: CT scan can help to detect L1 and L2 vertebral body invasion in patients with renal pelvis carcinoma. Early identification and optimal management of such patients is necessary.
\end{abstract}

Adult kidneys are located at the T12-L3 level lateral to the psoas muscle and vertebral body (1). The left and right kidney hilum lie at vertebral levels L1 and L2, respectively (1). Tumors originating in the renal pelvis can spread directly to

This article is freely accessible online.

Correspondence to: Chuang-Chi Liaw, MD, Division of HematoOncology, Department of Internal Medicine, Chang-Gung Memorial Hospital, 5, Fusing St., Gueishan Township, Taoyuan City, 333, Taiwan, R.O.C. Tel: +886 33281200 ext 8825, Fax: +886 3278211, e-mail: e102309@adm.cgmh.org.tw

Key Words: Renal pelvis carcinoma, Li, L2 vertebral bodies, bone metastasis. the regional areas along $\mathrm{L} 1$ and $\mathrm{L} 2$ and then grow into the vertebral bone. Bone metastasis occurs either via direct invasion of bone tissue or secondarily to bone marrow, with the latter being most common (2). Renal pelvis carcinoma involving the renal vein or inferior vena cava (IVC) has been linked to early-onset lung metastasis, as noted in our previous study (3). Direct invasion of renal pelvis carcinoma to L1 and L2 vertebral bodies may play a role in the pathogenesis of bone metastasis development. Computed tomographic (CT) scans of the abdomen are useful for tumor staging and can detect invasion of L1 and L2 vertebral bodies (4).

In this study, we retrospectively identified direct regional invasion of L1 and L2 from metastatic renal pelvis carcinoma based on CT scans. We hypothesized that regional tumors directly growing into L1 and L2 vertebral bodies in patients with renal pelvis carcinoma is linked to the development of bone metastases.

\section{Materials and Methods}

Study population. We conducted a retrospective case series study using data collected from patients with metastatic renal pelvis cancer admitted to the Oncology Ward of Chang-Gung Memorial Hospital, Taoyuan, Taiwan, between January 2010 and December 2017. A single medical oncologist specializing in urological cancer provided most of the data. All patients were hospitalized due to chemotherapy treatment and palliative care for complications. CT scans were performed in all cases to evaluate the extent of the tumor. The criteria were: (i) Metastatic renal pelvis carcinoma confirmed via pathology and CT scan, (ii) identification of direct invasion of L1 and L2 vertebral bodies from retrospective CT scan review, and (iii) detection of bone metastases using radionuclide images/CT scans. Representative CT scans of L1 and L2 vertebral body invasion are shown in Figure 1.

Evaluation. We next evaluated whether direct regional growth into L1 and L2 vertebral bodies from renal pelvis carcinoma is linked to 


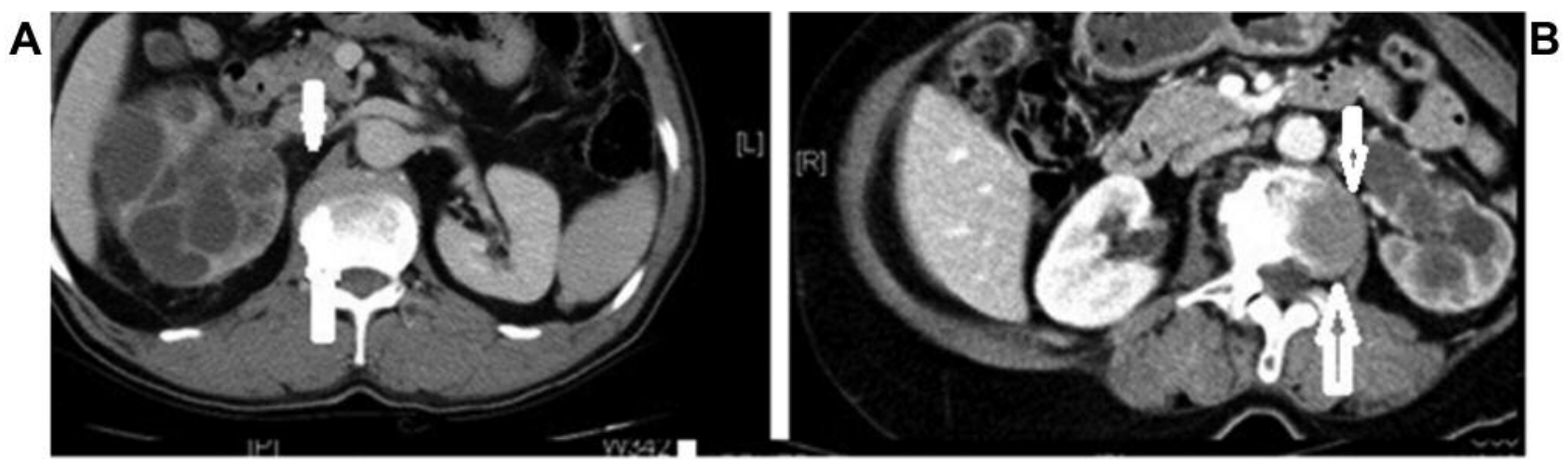

Figure 1. Computed tomographic scan showing direct L2 vertebral body invasion from renal pelvis carcinoma. Representative axial views of right (A) and left (B) renal pelvis malignancy showing tumors with direct L2 vertebral body invasion (arrows).

the development of bone metastases. Bone metastases were defined as the detection of metastatic bone lesions other than in the L1 and L2 vertebral bodies. Bone metastases were detected using CT scans in all patients and radionuclide images were used in 42 patients with 99mtechnetium-methylene diphosphonate (99mTc-MDP) whole-body bone scan. For staging, ${ }^{18} \mathrm{~F}$-fluorodeoxyglucose positron-emission tomography/CT $\left({ }^{18} \mathrm{~F}-\mathrm{FDG} \mathrm{PET} / \mathrm{CT}\right)$ was used in four cases. Evaluation for bone metastasis included initial L1 and L2 vertebral body invasion, initial renal vein/IVC involvement, suspicion of peritoneal spread, para-aortic lymph node (LN) metastases, and lung metastases.

Statistical analysis. Chi-squared test was used to detect differences between subgroups and differences with $p<0.05$ were considered statistically significant.

\section{Results}

This study included 71 consecutive patients with metastatic renal pelvis cancer. They consisted of 39 men and 32 women, aged between 39 and 88 years (median age $=65$ years). Of them, 24 patients did not undergo nephrourectomy procedures. Fifteen patients had multiple primary sites of urinary cancer, including five cases involving the bladder (one case simultaneously), nine cases involving the ureter (six cases simultaneously), and one case involving the bladder and the ureter. One case had undergone previous renal transplantation and one case had end-stage renal disease.

The patients' clinical characteristics are shown in Table I. The most common pathology in this study was urothelial carcinoma. Initial L1 and L2 vertebral body invasion were detected in $45(63 \%)$ patients; all presented with various degrees of back and radicular pain. Initial renal vein/IVC involvement was found in $43(61 \%)$ patients and lung metastasis occurred in $44(62 \%)$.

Detection of bone metastases other than L1 and L2 invasion from image examination, and pattern and location of bone metastases are shown in Table II. Of the 45 patients with initial L1 and L2 vertebral body invasion, invasion in all was detected
Table I. Clinical characteristics of 71 patients with metastatic renal pelvis carcinoma.

\begin{tabular}{|c|c|}
\hline Characteristic & Value \\
\hline \multicolumn{2}{|l|}{ Age, years } \\
\hline Median (range) & $65(39-88)$ \\
\hline \multicolumn{2}{|l|}{ Gender, $\mathrm{n}(\%)$} \\
\hline Male/female & $39 / 32$ \\
\hline \multicolumn{2}{|l|}{ Histology/cytology, n (\%) } \\
\hline Urothelial carcinoma & $67(94)$ \\
\hline Squamous cell carcinoma & $3(4)$ \\
\hline Lymphoepithelial-like carcinoma & $1(1)$ \\
\hline \multicolumn{2}{|l|}{ Initial tumor spread, n (\%) } \\
\hline Lung & $44(62)$ \\
\hline Liver & $7(10)$ \\
\hline \multicolumn{2}{|l|}{ Invasion, $\mathrm{n}(\%)$} \\
\hline Direct lumbar vertebral body & $45(63)$ \\
\hline Bone (other than L1 and L2) & $11(14)$ \\
\hline Adrenal gland & $3(4)$ \\
\hline \multicolumn{2}{|l|}{ Supraclavicular LNs, n (\%) } \\
\hline Yes & $5(7)$ \\
\hline \multicolumn{2}{|l|}{ CNS, n (\%) } \\
\hline Yes & $3(4)$ \\
\hline \multicolumn{2}{|l|}{ Para-aortic LN involvement, n (\%) } \\
\hline Yes & $36(51)$ \\
\hline \multicolumn{2}{|l|}{ Suspected peritoneal spread, n (\%) } \\
\hline Yes & $68(96)$ \\
\hline \multicolumn{2}{|l|}{ Renal vein/IVC involvement, $\mathrm{n}(\%)$} \\
\hline Yes & $43(61)$ \\
\hline
\end{tabular}

LN: Lymph node; CNS: central nervous system; IVC: inferior vena cava.

from retrospective CT scan review. Detection method included CT scan only in $21(66 \%)$ patients (one initially) and a combination of CT scan with radionuclide imaging in 11 (10 initially). A total of 11 cases was positive for radionuclide image studies, including 10 bone scans and one PET/CT. Ten out of 42 patients were positive for bone scans. PET/CT scans were checked in four patients and one was positive for bone 
Table II. Characteristics of 45 patients with direct lumbar vertebral body invasion or bone metastases from renal pelvis carcinoma.

\begin{tabular}{lc}
\hline Characteristic & No. of patients (\%) \\
\hline Presence of direct lumbar vertebral body invasion & 45 \\
Yes & $32(71)$ \\
Detection of bone metastasis other than L1 and L2 invasion & $13(29)$ \\
Yes & $21(66)$ \\
No & $0(0)$ \\
Method of image for diagnosis of bone metastases & $11(34)$ \\
CT scan only & 13 \\
Bone (or PET) scan only & $9(69)$ \\
CT scan + bone (or PET) scan & $4(21)$ \\
No demonstration of bone metastasis & $32(100)$ \\
Further image study not available & $0(0)$ \\
Probably tumor regression after chemotherapy & \\
Pattern of bone metastasis & $20(63)$ \\
Osteolytic & $12(38)$ \\
Osteoblastic & \\
Location of bone metastasis & \\
Lumbar spine only & \\
Lumbar spine + other sites & \\
\hline
\end{tabular}

metastasis. All bone lesions found on CT scans were osteolytic. Bone metastatic sites of $22(65 \%)$ patients were located in the lumbar spine only.

CT scans showed direct lumbar vertebral body invasion from renal pelvis carcinoma (Figure 2A). PET-CT scan showed multiple bone metastases Figure 2B). A CT scan with an axial view of the right renal pelvis malignancy is shown in Figure 3A and a tumor with direct L2 vertebral body invasion in Figure 3B. CT scans also showed left scapular bone metastasis. In Figure 3C, a bone scan showed multiple bone metastases. Figure 4A shows a CT scan of L2 invasion from renal pelvis carcinoma. Disease progression following chemotherapy with an increase in L2 vertebral invasion and L3 vertebraI metastases can be seen in Figure $4 \mathrm{~B}$ and $\mathrm{C}$.

A total of 71 patients were evaluated for associations of factors of bone metastasis (Table III). We found that initial L1 and L2 vertebral body invasion $(p<0.00001)$ was associated with the development of bone metastases.

\section{Discussion}

The most common sites of metastasis from renal pelvis cancer are the LNs, bones, lungs, liver, and peritoneum (5, 6). Similarly to the literature, $48 \%$ of patients with metastatic renal pelvis carcinoma in this study had metastasis to bone. The spread of urological malignancies to bone is a poor prognostic factor. Early detection of metastatic bony lesions can improve quality of life with therapy (7).

Diagnosis of L1 and L2 vertebral body invasion and bone metastasis is possible based on symptoms, CT scan, and
Table III. Analysis of associations with bone metastasis in 71 patients with metastatic renal pelvis carcinoma.

\begin{tabular}{lcc}
\hline Total cases (n=71) & $\begin{array}{c}\text { Bone metastasis/ } \\
\text { total cases, } \mathrm{n}(\%) \\
32 / 71(45 \%)\end{array}$ & $p$-Value \\
& & \\
\hline $\begin{array}{l}\text { Direct lumbar vertebral body invasion } \\
\text { Yes }\end{array}$ & $32 / 45(71)$ & $<0.00001$ \\
$\quad$ No & $0 / 36(0)$ & \\
Suspected peritoneal spread & $31 / 68(46)$ & 0.68 \\
$\quad$ Yes & $1 / 3(33)$ & \\
$\quad$ No & & \\
Renal vein/IVC involvement & $21 / 43(49)$ & 0.43 \\
$\quad$ Yes & $11 / 28(39)$ & \\
$\quad$ No & $11 / 36(31)$ & 0.01 \\
Para-aortic LN involvement & $21 / 35(60)$ & \\
$\quad$ Yes & & \\
$\quad$ No & $18 / 44(41)$ & 0.37 \\
Lung metastasis & $14 / 27(52)$ & \\
$\quad$ Yes &
\end{tabular}

IVC: Inferior vena cava; LN: lymph node.

radionuclide images (8). The development of radicular pain, which is characterized by pain radiating along the dermatome of a nerve (9), as well as back pain, is related to invasion L1 and L2 vertebral bodies, which cause nerve root compression or irritation (3). CT scans are useful for the staging and detection of L1 and L2 vertebral body invasion and osteolytic bone metastasis $(4,10)$. However, initial L1 and L2 vertebral 

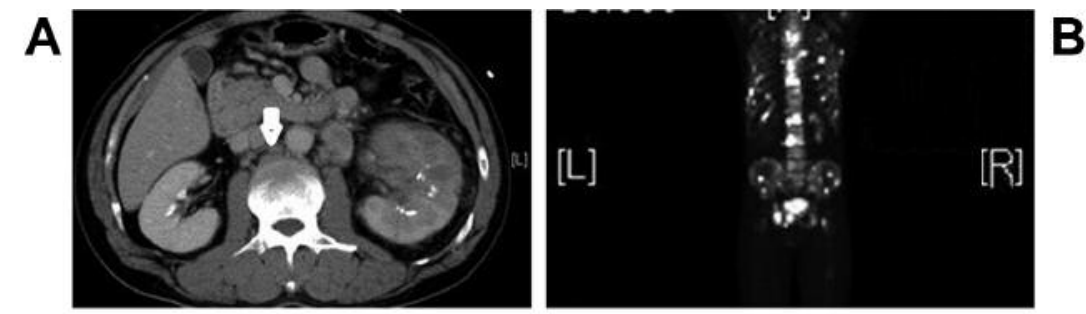

Figure 2. Computed tomographic scan showing direct lumbar vertebral body invasion from renal pelvis carcinoma. A: Axial view of left renal pelvis malignancy showing tumor with direct L2 vertebral body invasion (arrow). B: Positron-emission tomography-computed tomographic scan showing multiple bone metastases.
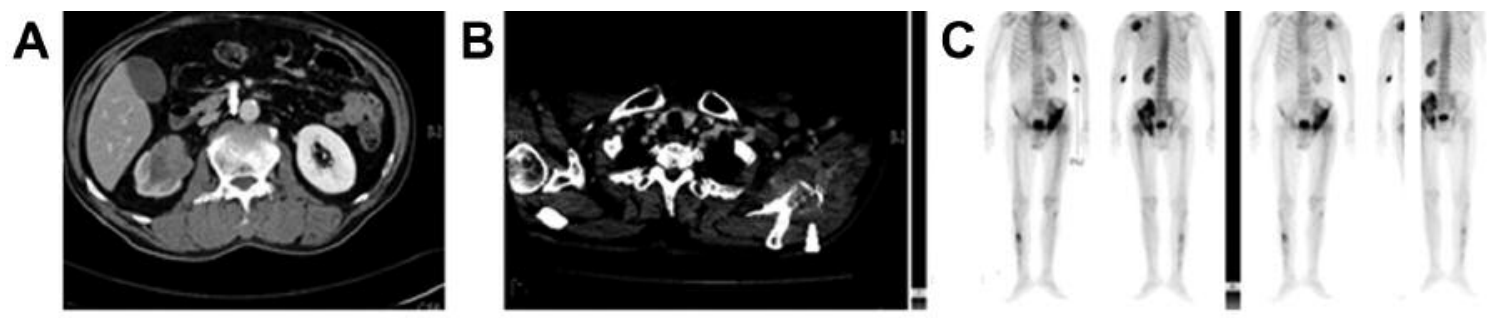

Figure 3. Computed tomographic scan showing direct lumbar vertebral body invasion from renal pelvis carcinoma. Axial view of right renal pelvis malignancy showing tumor with direct $L 2$ vertebral body invasion (A) and metastasis in the left scapular bone (B). (C) Bone scan showing multiple bone metastases. Arrows indicate tumors with vertebral body invasion. Arrowhead indicates left scapular bone destruction.
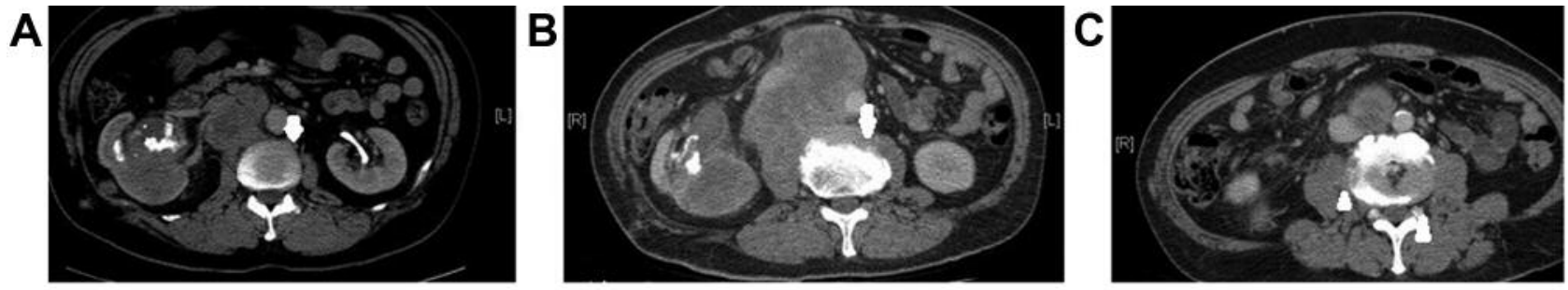

Figure 4. Computed tomographic scan showing direct lumbar vertebral body invasion from renal pelvis carcinoma. Axial view of right renal pelvis malignancy showing a tumor with direct invasion of L2 vertebral body (A), disease progression following chemotherapy with increased L2 vertebral body destruction $(B)$, and $L 3$ vertebral body metastases $(C)$. Arrows indicate tumors with vertebral body invasion. Arrowhead indicates $L 3$ vertebral body bone destruction.

body invasion is mainly detected from retrospective review of CT scans. ${ }^{99 \mathrm{~m}} \mathrm{Tc}-\mathrm{MDP}$ is commonly used as the bone scanning agent, whose uptake is related to osteoblastic activity in response to the cancer (10). Early bone invasion or the presence of some osteolytic metastases such as from multiple myeloma or hepatoma, can lead to false-negative bone scans (10-12). L1 and L2 vertebral body invasion are osteolytic lesions and can be identified from CT scan rather than from bone scan. ${ }^{18} \mathrm{~F}$-FDG PET/CT has a higher diagnostic value than whole-body ${ }^{99 \mathrm{~m}} \mathrm{Tc}-\mathrm{MDP}$ bone scan for the detection of osteolytic bone metastases, especially in the vertebrae $(12,13)$. PET/CT scans used in this study to detect the tumor extent only showed one case positive for bone metastasis.
Metastasis via a direct invasion of bone tissue is a mechanism for the development of bone metastases (2). We hypothesized that direct regional tumor growth into the L1 and L2 vertebral bodies in patients with renal pelvis carcinoma was linked to the development of bone metastases. Chemokines play a vital role in tumor progression and metastasis $(14,15)$, with the CXC chemokine ligand 12-CXC chemokine receptor type 4 (CXCL12-CXCR4) axis playing a pivotal role in bone metastasis (14-16). The bone microenvironment also plays an important role in the development of bone metastases and tumor progression $(17,18)$. Expression of inflammatory mediators that are critical for cancer growth is increased in bone metastasis (19). Only initial invasion of L1 and L2 
vertebral bodies was a factor associated with the development of bone metastases in our study.

There were several limitations to this study. Firstly, the case series data were collected retrospectively. Secondly, the diagnoses of L1 and L2 vertebral body invasion and bone metastases were based only on images and were not confirmed by pathology.

In conclusion, initial L1 and L2 vertebral body invasion was a factor significantly associated with the development of bone metastases. An initial CT scan can help detect L1 and L2 vertebral body invasion, but this feature can easily be overlooked. Early identification and optimal management of these patients is necessary.

\section{Conflicts of Interest}

There are no conflicts of interest regarding this study.

\section{Authors' Contributions}

L T-Y performed data analysis and manuscript writing/editing; L C$\mathrm{C}$ and $\mathrm{T}$ K-H contributed cases and management, and $\mathrm{J} \mathrm{Y}-\mathrm{H}$ contributed imaging knowledge. All Authors read and provided final approval of the manuscript.

\section{Acknowledgements}

The Authors wish to thank the oncology nursing staff for providing the best possible care for the patients of this study. We would like to thank Uni-edit (www.uni-edit.net) for editing and proofreading this manuscript.

\section{References}

1 Anderson JK and Cadeddu JA: Surgical anatomy of the retroperitoneum, adrenals, kidneys, and ureters. In: Campbell-Walsh Urology (10th edition). Wein AJ, Kavoussi LR, Novick AC, Partin AW and Peters CA: Philadelphia: Saunders Elsevier, pp 3-6, 2012.

2 Schmid-Alliana A, Schmid-Antomarchi H, Al-Sahlanee R, Lagadec P, Scimeca JC and Verron E: Understanding the progression of bone metastases to identify novel therapeutic targets. Int J MoI Sci 19(1): Pii:E148, 2018. PMID: 29300334. DOI: $10.3390 / \mathrm{ijms} 19010148$

3 Liao TY, Liaw CC, Tsui KH and Juan YH: Renal pelvis carcinoma with renal vein or inferior vena cava involvement linked to early-onset lung metastasis based on CT scan diagnosis. Anticancer Res 38(5): 3187-3192. 2018. PMID: 29715161. DOI: 10.21873 /anticanres.12583

4 Buckley JA, Urban BA, Soyer P, Scherrer A and Fishman EK: Transitional cell carcinoma of the renal pelvis: A retrospective look at CT staging with pathologic correlation. Radiology 201(1): 194198, 1996. PMID: 8816543. DOI: 10.1148/ radiology.201.1.8816543

5 Shinagare AB, Fennessy FM, Ramaiya NH and Jagannathanetal JP: Urothelial cancers of the upper urinary tract: Metastatic pattern and its correlation with tumor histopathology and location. J Comput Assist Tomogr 35(2): 217-222, 2011. PMID: 21412093. DOI: 10.1097/RCT.0b013e31820d7a37
6 Tan LB, Chang LL, Cheng KI, Huang $\mathrm{CH}$ and Kwan AL: Transitional cell carcinomas of the renal pelvis and the ureter: Comparative demographic characteristics, pathological grade and stage and 5-year survival in a Taiwanese population. BJU Int 103(3): 312-316, 2009. PMID: 18782312. DOI: 10.1111/ j.1464-410X.2008.07985.x

7 Rajarubendra N, Bolton D and Lawrentschuk N: Diagnosis of bone metastases in urological malignancies: An update. Urology 76(4): 782-790, 2010. PMID: 20346492. DOI: 10.1016/ j.urology.2009.12.050

8 O'Sullivan GJ and Carty FL: Cronin CG: Imaging of bone metastasis: An update. World J Radio 7(8): 202, 2015. PMID: 26339464. DOI: 10.4329/wjr.v7.i8.202

9 Govind J: Lumbar radicular pain. Aust Fam Physician 33(6): 409-412, 2004. PMID: 15253601.

10 Britton KE: Nuclear medicine imaging in bone metastases. Cancer Imaging 2(2): 84-86, 2002. DOI: 10.1102/1470-7330. 2002.0004

11 Chen CY, Wu K, Lin WH, Lan TY, Wang SY, Sun JS, Weng PW, Yen RF and Yang RS: High false-negative rate of Tc-99m MDP whole-body bone scintigraphy in detecting skeletal metastases for patients with hepatoma. J Formos Med Assoc 111(3): 140146, 2012. PMID: 22423667. DOI: 10.1016/j.jfma.2011.01.005

12 Ozülker T, Küçüköz UA, Ozülker F, Ozpaçac T: Comparison of ${ }^{18}$ F-FDG-PET/CT with $99 \mathrm{mTc}-\mathrm{MDP}$ bone scintigraphy for the detection of bone metastases in cancer patients. Nucl Med Commun 31(6): 597-603, 2010. PMID: 20224457. DOI: 10.1097/MNM.0b013e328338e909

13 Lin Z, Chen L, Xie Q, Zhang Y, Cheng L, Li H and Wang J: A comparative study of ${ }^{18} \mathrm{~F}$-fluorodeoxyglucose positron-emission tomography/computed tomography and $99 \mathrm{mTc}-\mathrm{MDP}$ wholebody bone scanning for imaging osteolytic bone metastases. BMC Med Imaging 15: 7, 2015. PMID: 25885599. DOI: 10.1186/s12880-015-0047-2

14 Sarvaiya PJ, Guo D, Ulasov I, Gabikian K and Lesniak MS: Chemokines in tumor progression and metastasis. Oncotarget 4(12): 2171-2185: 2013. PMID: 24259307. DOI: 10.18632/ oncotarget. 1426

15 Patel LR, Camacho DF, Shiozawa Y, Pienta KJ and Taichman RS: Mechanisms of cancer cell metastasis to the bone: A multistep process. Future Oncol 7(11): 1285-1297, 2011. PMID: 22044203. DOI: $10.2217 /$ fon. 11.112

16 Wang J, Loberg R and Taichman RS: The pivotal role of CXCL12 (SDF-1)/CXCR4 axis in bone metastasis. Cancer Metastasis Rev 25(4): 573-587, 2006. PMID: 17165132. DOI: 10.1007/s 10555-006-9019-x

17 Zheng Y, Zhou H, Dunstan RC, Sutherland RL and Seibel MJ: The role of the bone microenvironment in skeletal metastasis. J Bone Oncol 2(1): 47-57. 2012. PMID: 26909265. DOI: 10.1016/j.jbo.2012.11.002

18 Holen I: The bone microenvironment: multiple players involved in cancer progression. J Bone OncoI 5(3): 87-89, 2016. PMID: 27761362. DOI: $10.1016 /$ j.jbo.2016.05.004

19 Roca $\mathrm{H}$ and McCauley LK: Inflammation and skeletal metastasis. Bone Key Rep 4: 706, 2015. PMID: 26131358. DOI: 10.1038/bonekey.2015.75 